\title{
Geo-Engineering in Lakes: A Crisis of Confidence?
}

Bryan M. Spears, ${ }^{* \dagger}{ }^{\dagger}$ Stephen C. Maberly, ${ }^{\dagger}$ Gang Pan, ${ }^{\S}$ Ellie Mackay, ${ }^{\ddagger}$ Andy Bruere, ${ }^{\|}$Nicholas Corker, ${ }^{\perp}$ Grant Douglas," Sara Egemose, ${ }^{\otimes}$ David Hamilton, ${ }^{\nabla}$ Tristan Hatton-Ellis, ${ }^{O}$ Brian Huser, ${ }^{\diamond}$ Wei Li, ${ }^{\text {II }}$ Sebastian Meis, ${ }^{\not}$ Brian Moss, ${ }^{\star}$ Miquel Lürling, ${ }^{\rightarrow}$ Geoff Phillips, $^{+}$Said Yasseri, ${ }^{\infty}$ and Kasper Reitzel ${ }^{\otimes}$

${ }^{\dagger}$ Centre for Ecology \& Hydrology, Penicuik, Midlothian, EH26 0QB, U.K.

${ }^{\ddagger}$ Centre for Ecology \& Hydrology, Lancaster Environment Centre, Library Avenue, Bailrigg, Lancaster, LA1 4AP, U.K.

${ }^{\S}$ Research Center for Eco-Environmental Sciences, Chinese Academy of Sciences, 18 Shuangqing Road, Haidian District, Beijing 100085, China

"Bay of Plenty Regional Council, Rotorua, New Zealand

${ }^{\perp}$ Centre for Ecology \& Hydrology, Maclean Building, Benson Lane, Crowmarsh Gifford, Wallingford, Oxfordshire OX10 8BB, U.K.

\#CSIRO Land and Water, Private Bag 5, Wembley, Washington 6913, Australia

${ }^{\nabla}$ Environmental Research Institute, University of Waikato, Private Bag 3105, Hamilton 3240, New Zealand

${ }^{\circ}$ Natural Resources Wales, Maes-y-Ffynnon, Penrhosgarnedd, Bangor, Gwynedd, LL57 2DW, U.K.

- Swedish University of Agricultural Sciences, Department of Aquatic Sciences and Assessment, Box 7050, 75007 Uppsala, Sweden

II Wuhan Botanical Garden, Chinese Academy of Sciences, Moshan, Wuhan, P.R. China

"lanaplan GbR, Lobbericher Str. 5, 41334 Nettetal, Germany

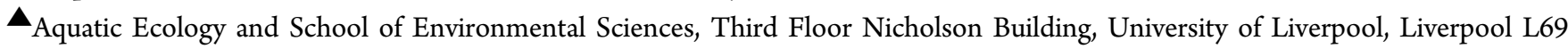
3GP, U.K.

`Water Quality Management Group, Department of Environmental Sciences, Wageningen University, P.O. Box 47, 6700 AA Wageningen, The Netherlands

${ }^{+}$Environment Agency, Kings Meadow House, Kings Meadow Road, Reading, RG1 8DQ U.K.

${ }^{\infty}$ Institut Dr. Nowak, Mayenbrook 1, D-28870 Ottersberg, Germany

${ }^{\otimes}$ Department of Biology, University of Southern Denmark, Campusvej 55, DK-5230 Odense M, Denmark

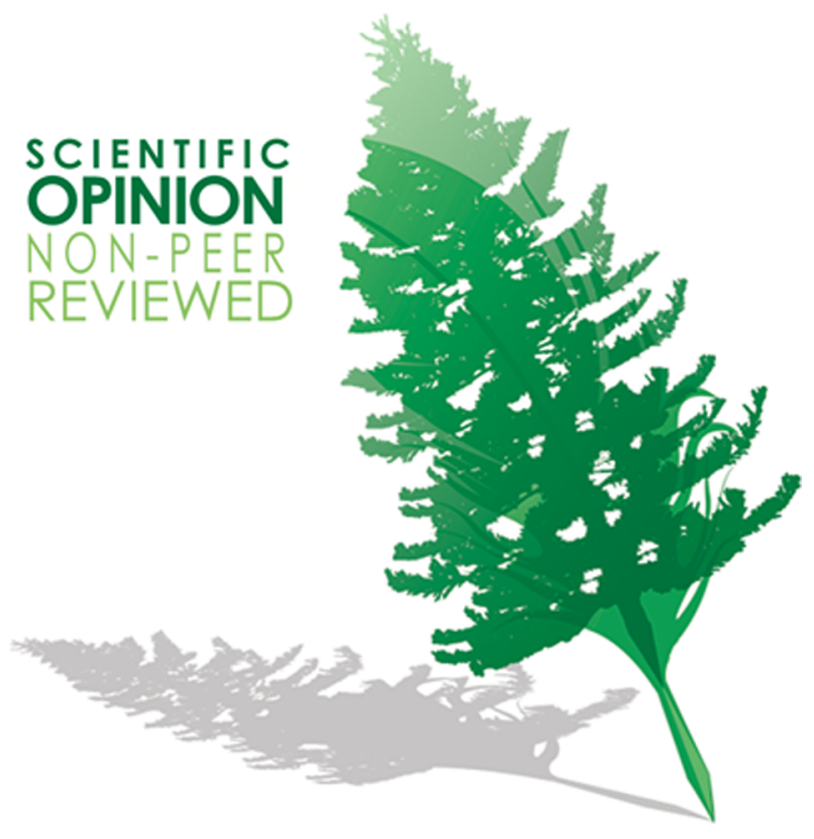

INTRODUCTION

The effective management of lakes suffering from eutrophication is confounded by a mosaic of interactions and feedbacks that are difficult to manipulate. For example, in lake processes can delay the relinquishment of legacy phosphorus (P) manifested within bed sediments for decades, even after effective catchment management. This recovery time is often deemed unacceptable and researchers have explored many inlake management measures designed to "speed-up" recovery. The manipulation of biogeochemical processes (commonly targeting P) using materials to achieve a desired chemical and/ or ecological response has been termed geo-engineering in lakes, and is becoming a commonly considered eutrophication management tool (Figure 1). Although this approach has been employed for many years it remains contentious largely due to variable results reported in the literature. This uncertainty risks ineffective management based on poorly designed or inappropriate applications. To address this, it is important that current levels of confidence in the approach be effectively communicated and that methods of increasing confidence are clearly demonstrated. We draw here on experiences of researchers and water managers at a global scale to demonstrate recent advances and consensus on recommendations (numbered below) for best practice. This information, although vital to underpinning successful management, has not been available in the peer reviewed literature.

Received: July 25, 2014

Published: August 19, 2014 


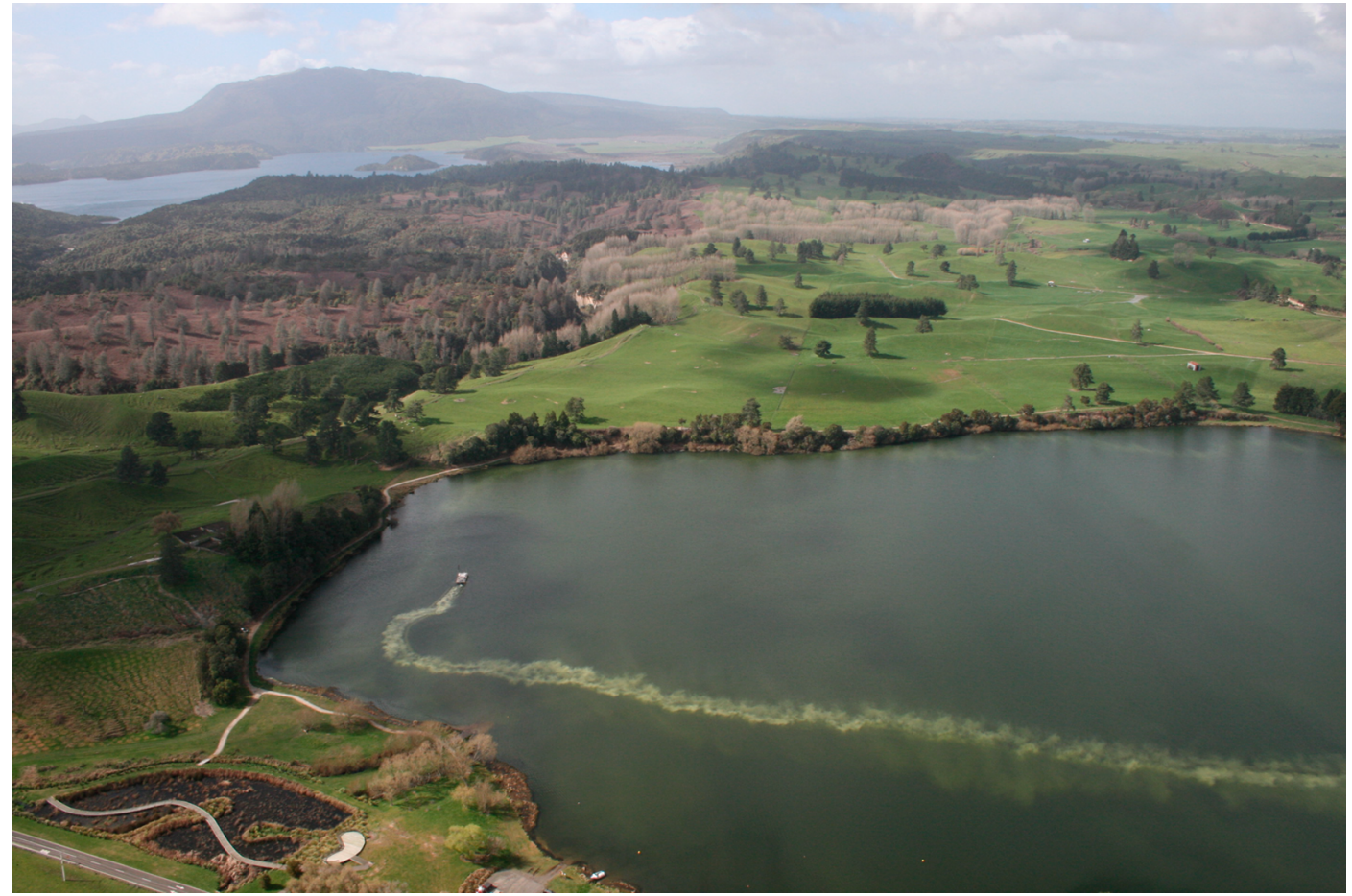

Figure 1. Application of Aqual-P to Lake Okaro, New Zealand, August, 2009.

\section{THE USE OF HIGH-FREQUENCY MONITORING OF TARGET PROCESSES CAN SUPPORT EFFECTIVE USE OF GEO-ENGINEERING}

Successful manipulation of biogeochemical processes requires comprehensive site specific understanding. Traditional seasonal, monthly, or even weekly monitoring of lakes can detect seasonal and long-term changes, but cannot capture the hourly to daily changes that frequently occur. High-frequency events (e.g., storms) can influence the effectiveness of geo-engineering materials through, for example, bed sediment disturbance associated with high winds and the delivery of high magnitude "pulses" of catchment nutrients following high rainfall events. Researchers now have access to near real-time data across a range of sentinel lakes documenting the effects of such events (e.g., http://www.gleon.org/). The power of high frequency monitoring has yet to be fully harnessed in the assessment of candidate lakes. In addition, monitoring systems when applied across multiple treated lakes will provide greater insight into responses at the meta-lake scale.

(1) Protocols should be developed combining traditional long-term data with high frequency monitoring programmes to provide the process-based understanding necessary to assess the candidacy of lakes for this approach.

(2) Evidence of responses following treatment should be combined across case studies to provide a meta-analysis against which the generality of responses in individual case studies can be assessed.

\section{THE DEVELOPMENT AND APPLICATION OF NEW MATERIALS HAS WIDENED THE SCOPE OF THE APPROACH}

The ideal amendment to lakes will remain geomorphologically stable, will interact only with the physicochemical environment through target processes, will be cost-effective, and will not negatively interact with ecological processes. To this end, the development of materials for nutrient, and in particular Premoval, has recently focused on three types (1) aluminosilicate minerals (e.g., modified zeolites), (2) existing catchment soils amended with natural materials (e.g., chitosan), and (3) mineral-based byproducts.

The use of aluminum $\left(\mathrm{Al} ; \mathrm{Al}_{2}\left(\mathrm{SO}_{4}\right)_{3}\right)$, and to a lesser extent iron $\left(\mathrm{Fe} ; \mathrm{Fe}_{5} \mathrm{O}_{4}\right)$, based salts has been relatively common in North America and parts of Europe. ${ }^{1}$ In the case of $\mathrm{Al}$ applications, data from multiple case studies are available and form the basis of the evidence in this field. More recently the clay mineral in most widespread use has been Phoslock, a lanthanum-modified bentonite which was specifically developed as a P-absorbent. ${ }^{2}$ However, other P-absorbent clays (e.g., hydrotalcite-clay nanohybrid) have recently been developed to provide more generic anion-exchange potential. Many mineral byproducts and catchment soils, by virtue of their often high $\mathrm{Ca}$ and/or $\mathrm{Fe}$ content represent attractive alternatives. In an attempt to limit the impact of exogenous materials on lake ecosystems, researchers in China have applied local soils modified with naturally occurring flocculants (e.g., chitosan) to lakes to disrupt both cyanobacterial blooms and sediment nutrient release, leading to recovery in submerged macrophytes. $^{3}$ However, successful macrophyte recovery will be dependent on the existence of macrophyte propagules in the lake bed or local soils. Additives including macrophyte propagules and oxygen nanobubbles are being developed to enhance ecological recovery further. There is an ever increasing arsenal of geo-engineering materials available to water managers; however, comprehensive understanding of their behavior in receiving waters is limited.

(3) An assessment of all reported target and nontarget effects associated with all available products should be conducted. 
(4) Decision support systems should be designed to assist material selection across a range of receiving water types.

\section{BETTER UNDERSTANDING OF PRODUCT-ENVIRONMENT INTERACTIONS CAN MITIGATE NONTARGET EFFECTS}

The operational performance of geo-engineering materials can be retarded by a range of physicochemical conditions in receiving waters. This can lead to potential ecotoxicological effects, reduction in operational performance, and alteration of coupled biogeochemical cycles (e.g., carbon and nitrogen). A range of analytical techniques has been applied to develop evidence with which these effects can be mitigated. Chemical equilibrium modeling has been demonstrated to be a useful tool for the prediction of metal leaching from materials. ${ }^{4}$ Sequential sediment extractions continue to provide the necessary, standardized information on site specific material-P binding ratios. ${ }^{1,5}$ Powerful analytical techniques (e.g., X-ray absorption spectroscopy) are being utilized to provide insights into specific binding mechanisms of the materials with the target chemical species in the presence of confounding constituents (e.g., humic materials). Collectively, these studies highlight the importance of humic substances, $\mathrm{Si}, \mathrm{pH}$, alkalinity, and material aging processes in receiving waters and sediments in reducing the binding efficiency and geomorphological stability of various materials.

(5) Standard protocols for dose determination should be developed based on published literature ${ }^{5}$ and knowledge of the likely behavior of the amendment material in the receiving water.

(6) The application of materials should be assessed in the context of "dose-response" (i.e., repeated smaller doses) as opposed to the common "single pill" (i.e., single large dose) approach to minimize the risk of unintended consequences.

Geo-engineering in lakes offers flexibility in the management of multiple pressures. However, researchers and water managers must work together to increase confidence in the application of this approach. We hope that our recommendations will help to increase the likelihood of management success and limit the risk of potentially costly unintended consequences.

\section{AUTHOR INFORMATION}

\section{Corresponding Author}

*E-mail: spear@ceh.ac.uk.

\section{Notes}

The authors declare no competing financial interest.

\section{REFERENCES}

(1) Huser, B. J.; Pilgrim, K. M. A simple model for predicting aluminum bound phosphorus formation and internal loading reduction in lakes after aluminum addition to lake sediment. Water Res. 2014, 53, 378-385.

(2) Robb, M. R.; Greenop, B.; Goss, Z.; Douglas, G. B.; Adeney, J. A. Application of Phoslock, an innovative phosphorus binding clay, to two Western Australian waterways - preliminary findings. Hydrol Process. 2003, 494, 237-243.

(3) Pan, G.; Yang, B.; Wang, D.; Gen, H.; Tian, B.; Zhang, M.; Yuan, $\mathrm{X}$.; Chen, J. In-lake algal bloom removal and submerged vegetation restoration using modified local soils. Ecol. Eng. 2011, 37, 302-308.

(4) Spears, B. M.; Lürling, M.; Yasseri, S.; Castro-Castellon, A. T.; Gibbs, M.; Meis, S.; McDonald, C.; McIntosh, J.; Sleep, D.; Van
Oosterhout, F. Lake responses following lanthanum-modified bentonite clay (Phoslock $囚)$ application: an analysis of water column lanthanum data from 16 case study lakes. Water Res. 2013, 47, 593042.

(5) Reitzel, K.; Hansen, J.; Andersen, F. O.; Hansen, K. S.; Jensen, H. $S$. Lake restoration by dosing aluminum relative to mobile phosphorus in the sediment. Environ. Sci. Technol. 2005, 39 (11), 4134-4140. 